\title{
Exploring, Documenting, and Improving Humanitarian Service Learning through Engineers Without Borders USA
}

\section{Dr. Tina Lee, University of Wisconsin-Stout}

Dr. Tina Lee is an Associate Professor of Anthropology and the Program Director for the Applied Social Science Program at the University of Wisconsin-Stout.

\section{Dr. Devin R. Berg, University of Wisconsin, Stout}

Devin Berg is an Associate Professor and Program Director of the B.S. Mechanical Engineering program in the Engineering and Technology Department at the University of Wisconsin - Stout.

\section{Dr. Elizabeth Anne Buchanan, UW-Stout}

Elizabeth Buchanan is Endowed Chair in Ethics and Acting Director, Office of Research and Sponsored Programs, at the University of Wisconsin-Stout. 


\title{
Exploring, Documenting, and Improving Humanitarian Service Learning through Engineers Without Borders USA
}

\begin{abstract}
Exploring, Documenting, and Improving Humanitarian Service Learning through Engineers without Borders-USA is a four-year project exploring a variety of challenges and opportunities in university-based service learning programs. Specifically, this project looks holistically at the inception and evolution of a new Engineers Without Borders USA chapter, while analyzing characteristics, values, and demographics of individuals involved in EWB community-based humanitarian projects in multiple chapters. Further, it examines the social, cultural, and professional interactions and exchanges between and among EWB members and community stakeholders in EWB projects, examining several projects from a variety of chapters across the country.
\end{abstract}

\section{Introduction}

Our project, "Exploring, Documenting, and Improving Humanitarian Service Learning through Engineers without Borders-USA," explores how engineering and technology students experience service learning during their undergraduate programs. ${ }^{1}$ One example of service learning is Engineers Without Borders (EWB) USA, which seeks to find practical solutions to community concerns, such as clean water or sustainable sewage systems. This project is studying a newly created EWB chapter working in Nicaragua to develop clean water systems, while understanding the needs and realities of life for both the citizens of the community and the volunteers. The project will unfold over four years and includes both engineering and social science students in the work.

Many universities and colleges offer such experiences to their students and believe they are contributing a valuable service to developing communities. ${ }^{2}$ However, service-learning projects are often compromised because students graduate, and/or faculty leaders move to other institutions, and community ties are lost. The project seeks to find best practices for university/college-based service learning for all stakeholders in these enterprises. This project is a collaboration between the professional society, Engineers Without Borders USA, and University of Wisconsin-Stout (UW-Stout) researchers, teachers, and students. Student attitudes, beliefs, and goals about community involvement are explored in a cross-institutional manner: In addition to 
studying the UW-Stout EWB chapter, the project looks at data collected by Engineers Without Borders USA and examines trends and practices throughout chapter projects.

In this poster presentation, we will present on activities to date, focusing on preliminary qualitative results. Both student and professional members of EWB chapters from across the US were interviewed about their experiences with and perspectives on their service work. In addition, members of the research team accompanied the UW-Madison chapter on an implementation/assessment trip to Ecuador. Areas under investigation included such issues as initial engagement, sustained participation, and professional responsibilities and ethical obligations of engineers. Participants also described their experiences with both positive and negative aspects of humanitarian service learning and offered thoughts on larger concerns surrounding professional responsibilities and ethics.

\section{Research Questions and Methods}

This project uses a mixed methods approach, including surveys, interviews, focus groups, content analyses, and field work to explore and seek answers to the following lines of inquiry:

1. Does participation in service learning such as EWB-USA contribute to a culture of ethical STEM practice? Do participants from service learning projects experience their STEM education in a qualitatively different way than those who do not?

2. How can we learn from the on-ground experiences of students and faculty to identify and promote best practices in humanitarian SL for a more ethically aware STEM culture?

(a) How does participation in SL "fit" into the typical practices of undergraduates? How interconnected is the social responsibility element of SL to the totality of their educational experiences?

(b) If students are not able to experience a project from inception to conclusion, including seeing the longer-term impacts of their work, are their experiences less meaningful? What are the professional responsibilities that students miss by participating in only some segments of a project?

(c) How can institutions encourage meaningful participation for faculty and students? What does that participation resemble, and what are the short and long-term effects of participation in international SL work through EWB-USA?

(d) How do we ensure participation in international SL activities on university campuses is driven by an ethical imperative and is a sustainable benefit for the communities being served?

(e) What institutional constraints face both faculty and student participants in EWB-USA chapters? What commonalities exist across institutions and what strategies can we develop to minimize such constraints for the betterment of SL?

3. Who is the primary client or beneficiary of SL? What is the balance between helping a community versus or contrasted to student experiences? 
The broader picture behind this research endeavor and a more in-depth look at the methodology has been presented previously. ${ }^{3}$

\section{Activities to Date}

To contextualize this project, we began by reviewing and coding thousands of EWB project reports from the last 10 years. We sought to understand the factors that produce successful and unsuccessful projects, as well as patterns in which projects are pursued in the first place and which are abandoned.

Then, beginning in fall 2015, all engineering students taking the UW-Stout course Impacts of Engineering have participated in a pre- and post-survey, examining their values and ethical beliefs regarding professional responsibilities and humanitarian service learning work. This course introduces students to the engineering design process, explores "past and present impacts on people, society, and the environment," and examines "contemporary and emerging issues related to engineering." Survey questions measure attitudes and competencies surrounding ethics, sustainability, the need to include social and environmental factors in designs, and attitudes towards including pro-bono and international work in careers. Text response questions asked students to reflect on the course and how it impacted their thinking. A total of 214 pre-survey responses and 170 post-survey responses have been gathered over three semesters.

As a control group, we also surveyed the general student population about their ethics and values related to professional responsibilities. A total of 123 students responded to that survey. UW-Stout is a unique institution in which to conduct such comparative work. As a polytechnic, most students are studying to join a technical profession but in a variety of fields. Early, preliminary interpretations of these survey results have been presented and discussed previously. ${ }^{4,5}$ A more complete data analysis will be presented later.

The project team has also participated in two EWB-USA conferences, where in depth focus groups and interviews were conducted. A total of 20 one-on-one interviews have been conducted and 15 individuals participated in focus groups. These data are the focus of this poster presentation.

Finally, one of the project PIs and a student research assistant went on an implementation visit to Ecuador. We will be revealing more details about this aspect of the project in subsequent articles, but it is worth noting a few points. First, due to some of the institutional hurdles identified as common in service learning work, the UW-Stout chapter was unable to travel for its implementation visit; however, the neighboring Madison chapter welcomed our anthropologist and her student investigator on a trip. The purpose of this trip for the UW-Madison chapter was to both complete implementation of a water system and to conduct assessments for a wash station and river restoration project. 


\section{Preliminary Findings}

At the mid-point of the project, three major themes are notable, and they include the role and importance of:

- In-country NGO and community partnerships

- Ethical and cultural awareness, in particular the importance of ethics training

- Gender

In reviewing project reports and discussing projects with EWB members, it is becoming clear that technical and design issues rarely make the difference between successful and an unsuccessful projects. ${ }^{6}$ The process for creating and vetting designs among students, technical mentors, and EWB staff is very effective and to be commended. On the other hand, both student and professional EWB members did point out the role of strong in-country partners in their successful projects and, in some cases, a need to gather more information about NGO partners to ascertain their relationships to the communities that projects are to benefit. Strong relationships with a broad cross-section of community members is also sometimes an issue in projects. Several interviewees discussed situations in which "community" was taken as a given while significant divisions among community members and their different views about what community needs are present and how they should be met were not known until designs had been finalized. This is an understandable outcome related to logistics in most cases-trips to communities are brief and communication between trips is often limited and takes place with one or two community members who are likely to be relatively privileged. In addition, many EWB members understandably have little training in methodologies that would allow them to more comprehensively assess community structures and needs.

The importance of ethical and cultural awareness has also been much discussed among our research participants. During in-depth interviews, many students eloquently described how the experience of participating in an EWB project gave them a different view of the ethics involved in engineering designs and a greater awareness of how cultural differences impact projects. In particular, many students discussed the need to be humble and to not "swoop in" to a community assuming that they have the perfect solution.

Finally, an unexpected, but upon reflection, not surprising theme is the role of gender in EWB work. It is well-documented that engineering remains a fairly male dominated field, ${ }^{7}$ and there have been initiatives at many institutions, including Stout, to increase the participation of females in the field. In contrast, EWB events are about evenly attended by women and men, and women are leaders in many chapters. Several women, both students and professional members, noted that EWB has made the difference for them between staying in the field and moving to a different career path since there is more space for collaboration and a value placed on "soft skills" that are often coded as feminine. EWB thus can play an important role in diversifying the engineering profession. 


\section{Next Steps}

The project will continue to collect data from EWB members with a new line of inquiry, encouraged by our collaboration with the UW-Madison chapter. We will examine demographics and institutional profiles of members and chapters to delve deeper into the ethical challenges raised by and in service learning. For example, one of the institutional barriers the UW-Stout chapter has faced in implementing their project was financial; students could not afford the costs related to international travel. Furthermore, time constraints limited Stout students' ability to miss two to three weeks of work. However, UW Madison's chapter did not face the same constraints, and importantly, most if not all of the Madison students had "free time" due to the high numbers of Advanced Placement courses with which they entered the university. While comparing and contrasting chapters has always been a part of the study, we will look more critically at the "haves" and "have-nots" involved in service learning.

\section{Acknowledgments}

This work is supported by the National Science Foundation under Grant No. EEC-1540301. Any opinions, findings, and conclusions or recommendations expressed in this material are those of the authors and do not necessarily reflect the views of the National Science Foundation.

\section{References}

[1] Christine I Celio, Joseph Durlak, and Allison Dymnicki. A meta-analysis of the impact of service-learning on students. Journal of Experiential Education, 34(2):164-181, 2011.

[2] Jen Schneider, Juan Lucena, and Jon A Leydens. Engineering to help. IEEE Technology and Society Magazine, 28(4), 2009.

[3] Devin R. Berg, Tina Lee, and Elizabeth Buchanan. A methodology for exploring, documenting, and improving humanitarian service learning in the university. Journal of Humanitarian Engineering, 4(1), 2016. doi: 10.6084/m9.figshare.2758129.v2.

[4] Devin R. Berg and Tina Lee. Incorporation of liberal education into the engineering curriculum at a polytechnic. In 2016 ASEE Annual Conference \& Exposition, New Orleans, Louisiana, June 2016. ASEE Conferences. doi: 10.18260/p.25664. URL https: / / peer.asee.org/25664.

[5] Tom Lacksonen, Devin R. Berg, and Scott Springer. Global engineering projects from the Young African Leaders Initiative. In 2017 ASEE Annual Conference \& Exposition, Columbus, Ohio, June 2017. ASEE Conferences.

[6] Donna Riley. Engineering and social justice. Morgan \& Claypool Publishers, 2008. doi: 10.2200/S00117ED1V01Y200805ETS007.

[7] Joscha Legewie and Thomas A. DiPrete. The high school environment and the gender gap in science and engineering. Sociology of Education, 87(4):259-280, 2014. doi: 10.1177/0038040714547770. 